\title{
Methods to study the roles of Rho GTPases in platelet function
}

Francisco Rivero and Simon Calaminus

Centre for Atherothrombosis and Metabolic Disease, Hull York Medical School, Faculty of Health Sciences, University of Hull, Hull, United Kingdom

\section{Corresponding author}

Simon Calaminus

Centre for Atherothrombosis and Metabolic Disease

Hull York Medical School, Faculty of Health Sciences

University of Hull

Cottingham Road, HU6 7RX Hull

United Kingdom

Tel: 01482466798

Email: simon.calaminus@hyms.ac.uk

Running title: Rho-dependent processes in platelets 


\section{Summary}

Platelets are a critical cell for prevention of bleeding. Part of the response to the formation of the thrombus is the activation of the actin cytoskeleton, with an inability to effectively activate the cytoskeleton linked to thrombus formation defects and instability. The control of this process is linked to activation of the Rho GTPases, Cdc42, Rac1 and RhoA, although additional small GTPases such as Rif and Rap have been shown to play roles in platelet function.

Here we will describe the methodology to accurately understand how Rho GTPases are activated in platelets. Due to the technical limitations of working with platelets, such as their lack of ability to be transfected, the majority of work has been carried out either using inhibitors of Rho GTPases or within knock out mouse models. Studies can be conducted both in suspension samples and also in spread platelets. In suspension the platelets will undergo a shape change response, but will not be able to spread. In spread platelets it is possible to examine the effects of the matrix environment, such as concentration, type and stiffness on Rho GTPase function within platelet activation and platelet spreading.

Key Words: Actin, RhoA, Rac1, platelets, cAMP, cGMP, pulldown assay, Y27632, Rhosin A 


\section{Introduction}

Platelets are a blood cell critical to maintaining hemostasis. Too few platelets leads to a bleeding phenotype, whilst too many can lead to excessive thrombus formation and therefore heart attack or stroke. During thrombus formation the platelets undergo a coordinated activation process initiated by platelet agonists. This coordinated approach allows for the platelets to form a strong and effective thrombus that can resist the shear stress present within the blood vessel. A key part of this response is that the platelet can effectively rearrange its actin cytoskeleton and the production of fully or partially spread platelets, dependent on where they are in the thrombus.

Platelet spreading is a coordinated rearrangement of the platelet shape in response to the presence of an extracellular matrix protein. This spreading process is controlled by the activity of the Rho GTPases (1). There are over 20 small GTPases among mammalian cells, with differing expression patterns according to cell type (reviewed in ref. (2)). The most wellknown Rho GTPases, Cdc42, Rac, and RhoA, have been extensively researched and have been linked to formation of filopodia, lamellipodia and stress fibers respectively $(1,2)$. In platelets the role of Cdc42 is unclear. One study showed that platelets lacking Cdc42 do not form filopodia on fibrinogen and collagen related peptide (CRP), whilst a second study showed no effect on fibrinogen, but an inhibition of filopodia formation on von Willebrand factor $(3,4)$. Furthermore, Rif has also been proposed as a filopodia initiator, but Cdc42 may be able to compensate for its loss (5). However, Rac1 has been conclusively shown via chemical inhibition and knock out studies to control lamellipod formation in platelets (6). RhoA has been shown to control platelet shape change and stress fibre formation (7-9). In addition to filopodia, lamellipodia and stress fibers, the platelet also forms the actin nodule (10). This actin structure is thought to be similar to the podosome, as it is a circular actin structure dependent on the Arp2/3 complex and tyrosine phosphorylation, and has a ring of vinculin and integrins (11). The actin nodule is a dynamic structure that is thought to play a role in resistance of shear stress. Interestingly, the actin nodule is thought to be linked to filopodia and lamellipodia formation, as it disappears when stress fibers are formed.

In addition to the effects on platelet spreading, inhibition of either actin nodules, lamellipodia, or stress fiber formation has been shown to lead either to reduction of thrombus formation, and/or thrombus instability at arterial rates of shear $(8,11,12)$. Furthermore, a sustained 
cytoskeletal response is required, as post perfusion of Rac inhibitors or agents that lead to RhoA inhibition causes thrombus instability $(12,13)$.

The platelet spreading process can be followed in vitro. Initially, matrix proteins are attached to a glass coverslip. A number of platelet matrix proteins have been used including Horm collagen (a mixture of collagen I and III), fibrinogen, fibronectin, fibrin, von Willebrand factor (with or without ristocetin or botrocetin), laminin, the collagen-mimetic peptide GFOGER, CRP, oxidized low density lipoprotein (oxLDL), thrombin, and thrombospondin-1 (10, 14-20). The coverslips are then blocked with bovine serum albumin (BSA) before addition of the platelets. The platelets are spread for a designated time before fixation, and if required completion of immunofluorescence or proximity ligation. The coverslips are then mounted and imaged using differential interference contrast (DIC), conventional fluorescence, confocal or superresolution microscopy. Analysis of the images, can consist of analysis of surface area, numbers adhered, number of platelets with filopodia, proportion of cells with stress fibers or actin nodules, number of actin nodules per platelet, or the size of actin nodules. However, the extent of spreading can depend not only on the type of matrix protein but also its concentration, stiffness, and if it is combined with multiple other matrix proteins $(16,21$, 22).

Alongside analysis of fixed platelet samples, real time microscopy can also be conducted. This allows for dynamic understanding of the spreading process. Usually this is completed using DIC microscopy. However, with the production of Life-act platelets it is possible to follow the actin cytoskeleton in real time during the spreading process using fluorescence microscopy (11).

Although this assay allows for a global understanding of platelet spreading, it can also be used to investigate specific proteins, or for understanding cell signaling within the spreading process. In these cases, either knock out platelets or chemical inhibition of proteins can be used to understand a particular protein's role within spreading. These inhibitors can either be preincubated with platelets prior to spreading on matrix proteins, or alternatively platelets can be allowed to spread before the addition of inhibitors to fully activated and spread platelets. In addition to imaging, spread platelets can be lysed and the samples analyzed by Western blot. This then allows changes in the phosphorylation status of, for example, RhoA to be monitored with phosphor-specific antibodies, and changes in the levels of activated RhoA can biology, Vol. 1821, pp.199-217, Humana Press. The final authenticated version is available online at: https://doi.org/10.1007/978-1-4939-8612-5_14. 
be monitored using a classical activation (or pull-down) assay based on a fusion protein of glutathione-S-transferase (GST) and the Rho GTPase binding domain of effector molecules like Rhotekin.

\section{Materials}

\subsection{Platelet Isolation}

1. Acid Citrate Dextrose (ACD): $113.8 \mathrm{mM}$ glucose, $29.9 \mathrm{mM}$ trisodium citrate, $72.6 \mathrm{mM}$ $\mathrm{NaCl}$ and $2.9 \mathrm{mM}$ citric acid. Adjust $\mathrm{pH}$ to 6.4 .

2. $0.3 \mathrm{M}$ citric acid.

3. Modified Tyrode's buffer: $134 \mathrm{mM} \mathrm{NaCl}, 20 \mathrm{mM}$ HEPES, $0.34 \mathrm{mM} \mathrm{NaH}_{2} \mathrm{PO}_{4}, 12 \mathrm{mM}$ $\mathrm{NaHCO}_{3}, 2 \mathrm{mM} \mathrm{KCl}, 1 \mathrm{mM} \mathrm{MgCl}$ and $5.6 \mathrm{mM}$ glucose. Adjust pH to 7.3 .

4. Wash Buffer: $36 \mathrm{mM}$ citric acid, $10 \mathrm{mM}$ ethylendiaminotetracetic acid (EDTA), $5 \mathrm{mM}$ glucose, $5 \mathrm{mM} \mathrm{KCl}$ and $90 \mathrm{mM} \mathrm{NaCl}$. Adjust pH to 6.5 .

5. Bijou tubes.

6. Hypodermic eccentric luer Syringes (1-50-mL)

7. For human blood withdrawal use Vacutainer safety lok $21 \mathrm{G}$ butterfly needles.

8. For murine blood withdrawal use $25 \mathrm{G}$ needles

9. Single use tourniquet, sterile cotton wool, isopropyl wipes, spot plasters and disinfectant wipes.

10. Swingout centrifuge.

11. Beckman Coulter counter ( $\mathrm{Z}^{\mathrm{TM}}$ Series Coulter - Cell and Particle Counter) or hemocytometer.

\subsection{Platelet Spreading}

1. Glass coverslips (ø $12 \mathrm{~mm}$ ) and glass slides.

2. 6-well and 24-well plates (glass bottomed for real-time spreading)

3. Phosphate Buffered Saline (PBS), commercially available from various sources.

4. Matrix proteins (final concentrations):

a. $3-100 \mu \mathrm{g} / \mathrm{mL}$ fibrinogen (Enzyme Research) prepared fresh in PBS. 
b. $100 \mu \mathrm{g} / \mathrm{mL}$ Horm collagen (Takeda) prepared fresh from $1 \mathrm{mg} / \mathrm{mL}$ commercial stock in collagen diluent (provided).

c. $100 \mu \mathrm{g} / \mathrm{mL}$ fibronectin (Sigma) frozen aliquots prepared in PBS

d. $10-100 \mu \mathrm{g} / \mathrm{mL}$ oxLDL (own source) prepared fresh in PBS (see Note 1).

e. $10-100 \mu \mathrm{g} / \mathrm{mL}$ native LDL (own source) prepared fresh in PBS (see Note 1).

f. $\quad 10-100 \mu \mathrm{g} / \mathrm{mL}$ laminin (Sigma) frozen aliquots prepared in PBS.

5. Platelet agonists (final concentrations):

a. $0.1 \mathrm{U} / \mathrm{mL}$ thrombin diluted in PBS from $10 \mathrm{U} / \mathrm{mL}$ stock (see Note 2).

b. $100 \mu \mathrm{g} / \mathrm{mL}$ Horm collagen (Takeda) prepared fresh from $1 \mathrm{mg} / \mathrm{mL}$ commercial stock in collagen diluent (provided).

6. Platelet inhibitors (final concentrations):

a. $10-100 \mathrm{nM}$ prostacyclin (Cayman Chemicals) diluted in PBS; prepare a $2 \mathrm{mM}$ stock solution in methanol and store aliquots at $-20^{\circ} \mathrm{C}$ (see Note 3).

b. $10 \mathrm{nM}-1 \mu \mathrm{M}$ S-nitrosoglutathione (GSNO) (nitric oxide donor) diluted in PBS.

c. $2 \mathrm{U} / \mathrm{mL}$ apyrase diluted in PBS.

d. $10 \mu \mathrm{M}$ indomethacin diluted in PBS from stock; prepare a $10 \mathrm{mM}$ stock solution in dimethylsulfoxide (DMSO) (see Note 4).

7. Incubator $\left(37^{\circ} \mathrm{C}\right)$.

8. PBS-BSA: BSA that is depleted of fibrinogen, fibronectin and von Willebrand factor (Sigma) is dissolved in PBS to $5 \mathrm{mg} / \mathrm{mL}$ in a conical tube. The BSA is then denatured by placing the conical tube in boiling water for $10 \mathrm{~min}$, before being cooled on ice to room temperature, at which point it is filtered with a $0.45-\mu \mathrm{m}$ filter. After filtration the BSA can be stored frozen and the relevant amount defrosted for each experiment (see Note 5).

9. Rho GTPase inhibitors (final concentrations):

a. $50 \mu \mathrm{M}$ NSC23766 prepared in water and frozen as $50 \mathrm{mM}$ stock.

b. $10 \mu \mathrm{M}$ EHT1864 prepared in water and frozen as $10 \mathrm{mM}$ stock.

c. $10 \mu \mathrm{M}$ Y27632 prepared in water and frozen as $10 \mathrm{mM}$ stock.

d. $10 \mu \mathrm{M}$ RhosinA prepared in DMSO and frozen as $10 \mathrm{mM}$ stock.

10. Fixing solution: $4 \%$ paraformaldehyde, diluted in PBS from commercial $16 \%$ solution (Fisher).

11. Permeabilizing solution: $0.1 \%(\mathrm{v} / \mathrm{v})$ Triton-X-100 in PBS.

12. Fluorescein isothiocyanate (FITC)-labeled phalloidin (Sigma P5282) or tetramethylrhodamine isothiocyanate (TRITC)-labeled phalloidin (Sigma P1951): 
Dissolve to $0.1 \mathrm{mg} / \mathrm{mL}$ in methanol to obtain a $100 \mu \mathrm{g} / \mathrm{mL}$ stock solution. Store at $20^{\circ} \mathrm{C}$ protected from light (see Note 6 ).

13. Primary antibodies: Arp2/3 complex (p34 Arp2 subunit, Millipore), vinculin (clone hVIN-1, V9131 Sigma) and monoclonal anti-phosphotyrosine (clone 4G10, 05-1050 Millipore) can be used to label actin nodules. Primary antibodies specific for various other actin cytoskeleton components are available commercially or from researchers, as per the experimental requirements.

14. Secondary antibodies: choose from a variety of Alexa-coupled secondary antibodies (Invitrogen) depending on the host species of the primary antibody and the combination of fluorochromes required in the case of double staining.

15. Prolong Diamond antifade mounting medium (Thermo Scientific) or equivalent.

16. Conventional fluorescence microscope or confocal microscope equipped with $63 \times$ or 100x oil immersion lenses.

17. Inverted fluorescence microscope equipped with $63 \times$ or $100 \times$ oil immersion lenses and differential interference contrast (DIC) setup.

18. Image J: Available at https://imagej.nih.gov/ij/download.html.

\subsection{Westerns Blot and RhoA/Rac1 Activation Assay}

1. 6-well plates or $10-\mathrm{cm}$ plastic dishes.

2. PBS (see Subheading 2.2 item 3 )

3. PBS-BSA (see Subheading 2.2 item 8)

4. Matrix proteins (see Subheading 2.2 item 4)

5. Cell scrapers

6. Lysis buffer general - signalling (phosphorylation status of RhoA/Rac) (300 mM NaCl, 20 mM Tris, 2 mM EDTA, 2 mM EGTA, 2\% NP-40).

7. Protein concentration determination kit (Cytoskeleton).

8. $6 \times$ Laemmli buffer: $30 \%$ sodium dodecylsulfate (SDS), $4.7 \mathrm{~mL}$ of glycerol, $1.2 \mathrm{~mL}$ of $0.5 \mathrm{M}$ Tris- $\mathrm{HCl} \mathrm{pH} 6.8,6 \mathrm{mg}$ of bromophenol blue and $0.93 \mathrm{~g}$ of dithiothreitol (DTT) in $10 \mathrm{~mL}$ of distilled water.

9. Platelet agonists (see Subheading 2.2 item 5)

10. Platelet inhibitors (see Subheading 2.2 item 6)

11. Primary antibodies:
a. Phospho RhoA ${ }^{\text {Ser188 }}$ (Santa Cruz) 
b. Phospho Rac1 ${ }^{\text {Ser71 }}$ (Cell Signalling Technology)

C. RhoA (New England Biolabs).

d. Rac1 (New England Biolabs).

e. GAPDH (Millipore)

12. Secondary antibodies

a. Goat anti-mouse 800 (LiCor Biotechnology): use at 1:15,000 dilution.

b. Goat anti-mouse 680 (LiCor Biotechnology): use at 1:15,000 dilution.

13. Rac1 activation assay kit (Cytoskeleton). The kit contains lysis buffer and PAK-GST glutathione sepharose beads. Reconstitute beads in $500 \mu \mathrm{L}$ of sterile distilled water to yield a stock concentration of $1 \mathrm{mg} / \mathrm{mL}$. Store $20-\mu \mathrm{L}$ aliquots at $-80^{\circ} \mathrm{C}$.

14. RhoA activation assay kit (Cytoskeleton). The kit contains lysis buffer and RhotekinGST glutathione sepharose beads. Reconstitute beads in $500 \mu \mathrm{L}$ of sterile distilled water to yield a stock concentration of $1 \mathrm{mg} / \mathrm{mL}$. Store $20-\mu \mathrm{L}$ aliquots at $-80^{\circ} \mathrm{C}$.

15. Standard equipment, reagents and buffers for SDS-polyacrylamide gel electrophoresis (PAGE) and Western blot. For signal detection we favor a fluorescence-based system

\section{Methods}

\subsection{Platelet Isolation}

\subsubsection{Human platelet isolation}

1. Using standard venipuncture techniques collect whole blood from the vein of a healthy volunteer into a syringe containing ACD to achieve a ratio of 1:5 (ACD:blood). Mix the syringe gently to ensure $A C D$ is fully mixed with the blood (see Note 7).

2. Transfer the blood from syringe to $10-\mathrm{mL}$ bijou tubes.

3. Centrifuge the bijou tubes at $180 \times \mathrm{g}$ for $10 \mathrm{~min}$ at room temperature.

4. Transfer the top two thirds of the platelet rich plasma (PRP) into separate bijou tubes. 
5. The tubes containing the whole blood are then re-centrifuged at $200 \times \mathrm{g}$ for additional $10 \mathrm{~min}$ at room temperature.

6. The remaining PRP is then removed, trying not to disturb the buffy coat. This PRP is then combined with the PRP collected in step 4.

7. Adjust the $\mathrm{pH}$ of the collected PRP to 6.4 by adding $20 \mu \mathrm{L} / \mathrm{mL}$ of $0.3 \mathrm{M}$ citric acid. This $\mathrm{PRP}$ is then centrifuged at $650 \times \mathrm{g}$ for $12 \mathrm{~min}$ at room temperature.

8. The supernatant containing the platelet poor plasma (PPP) is discarded. Resuspend the platelet pellet gently in $3 \mathrm{~mL}$ of wash buffer (see Note 8).

9. Centrifuge the re-suspended pellets for additional $12 \mathrm{~min}$ at $1000 \times \mathrm{g}$ at room temperature.

10. Drain the supernatant and wipe the inside of the tube using a tissue. Care must be taken to not to disturb the pellet.

11. Resuspend the pellet in modified Tyrode's buffer.

12. Platelets are then incubated for $30 \mathrm{~min}$ at $37^{\circ} \mathrm{C}$ to rest before use.

13. During platelet resting obtain a platelet count using either a Beckman Coulter counter or a hemocytometer (see Note 9).

\subsubsection{Mouse platelet isolation}

1. Euthanize mouse according to appropriate ethical guidelines (see Note 10). Using scissors open the abdominal wall and gently push the intestines to your right hand side to expose the inferior vena cava (see Note 11).

2. Take blood from the inferior vena cava into a 1-mL syringe with a $25 \mathrm{G}$ needle containing $50 \mu \mathrm{L}$ of $\mathrm{ACD}$. 
3. Remove the needle and transfer blood into a microcentrifuge tube containing $200 \mu \mathrm{L}$ of Tyrode's buffer.

4. Centrifuge blood at $400 \times g$ for 6 min in a microcentrifuge.

5. Remove the top $700 \mu \mathrm{L}$ of blood from the tube.

6. Centrifuge the blood at $200 \times \mathrm{g}$ for $6 \mathrm{~min}$ in a swingout centrifuge.

7. Remove the PRP from the blood (see Note 12) and transfer to a fresh tube.

8. If needed add $200 \mu \mathrm{L}$ of Tyrode's buffer to the blood and repeat steps 6 and 7 .

9. Add either $1 \mu \mathrm{L}$ of $2 \mathrm{mM} \mathrm{PGI}_{2}$ or $20 \mu \mathrm{L}$ of $0.3 \mathrm{M}$ citric acid to the PRP and centrifuge at $1000 \times g$ for 6 min in a swingout centrifuge.

10. Remove the plasma, resuspend the platelets in Tyrode's buffer and count using either a Beckman Coulter counter or a hemocytometer.

\subsection{Platelet Spreading for Imaging Analysis (Fixed)}

1. Place 12-mm glass coverslips into the relevant number of wells in a 24-well plate.

2. Coat the coverslips by pipetting up to $200 \mu \mathrm{L}$ of the matrix protein of choice onto the coverslips within the wells. Prepare a BSA-only control at this point (have one coverslip to which PBS-BSA is added). The matrix protein will be held in place by surface tension on the coverslip and so will not cover the whole of the well. Cover the 24-well plate with the lid and incubate at $4^{\circ} \mathrm{C}$ overnight (see Note 13).

3. On the day of use prepare a minimum of $500 \mathrm{~mL}$ of PBS for washing coverslips and turn on incubator and set to $37^{\circ} \mathrm{C}$. 
4. Remove the plates from the fridge and remove the matrix protein solution. Then wash the coverslips twice with $500 \mu \mathrm{L}$ of PBS to remove any unbound matrix protein using a Pasteur pipette or an aspiration device. Leave the plates covered with PBS.

5. Block the coverslips with $300 \mu \mathrm{L}$ of PBS-BSA for $1 \mathrm{~h}$ at room temperature.

6. Remove the PBS-BSA solution and then wash the coverslips twice with $500 \mu \mathrm{L}$ of PBS to remove any unbound BSA using a Pasteur pipette or aspiration device.

7. At this point resuspend washed platelets to $2 \times 10^{7} / \mathrm{mL}$ in Tyrode's buffer. There are now two different methods that can be followed.

\subsubsection{Prior incubation of inhibitors method}

1. Add Rho GTPase inhibitors to the platelet suspension at the correct concentration (see Subheading 2.2 item 9). Preincubate the inhibitors with the platelets for 2 min prior to addition to the coverslips.

2. Pipette $200 \mu \mathrm{L}$ of platelet suspension onto each coverslip. Place the lid on the 24well plate, and then place the 24 -well plate in the $37^{\circ} \mathrm{C}$ incubator for $45 \mathrm{~min}$ to allow platelets to adhere to the coverslips.

3. After 45 min take out the plate from the incubator and remove the solution. Wash the coverslips twice with $500 \mu \mathrm{L}$ of PBS to remove any unbound platelets using a Pasteur pipette or aspiration device.

4. After washing place the 24-well plate in a fume hood and add $300 \mu \mathrm{L}$ of fixing solution onto each of the coverslips and leave for $10 \mathrm{~min}$ at room temperature to fix the adhered platelets.

5. After $10 \mathrm{~min}$, still in the fume cupboard, remove the fixing solution and wash the coverslips twice with $500 \mu \mathrm{L}$ of PBS to remove any excess paraformaldehyde using a Pasteur pipette or aspiration device (see Note 14). 
6. To permeabilize the platelet cell membrane, remove the PBS and pipette $200 \mu \mathrm{L}$ of permeabilizing solution onto the wells and incubate for $5 \mathrm{~min}$ at room temperature-

7. Remove the permeabilizing solution with a Pasteur pipette or aspiration device and then wash coverslips twice with $500 \mu \mathrm{L}$ of PBS to remove any excess Triton X-100.

8. If only staining of the actin cytoskeleton is needed proceed to step 10. Otherwise, to complete antibody staining prepare the correct antibody mix and concentration in PBS. Then remove the PBS and add $200 \mu \mathrm{L}$ of antibody mix to each coverslip for the appropriate incubation time (see Note 15).

9. Remove the antibody mix from the coverslips and then wash twice with $500 \mu \mathrm{L}$ of PBS with a Pasteur pipette.

10. To identify the actin cytoskeleton a phalloidin staining can be performed: prepare a 1:1000 dilution of FITC or TRITC-phalloidin in PBS. Otherwise to complete the antibody staining alongside staining for the actin cytoskeleton, remove the PBS of the last wash step. Prepare a 1:1000 dilution of FITC or TRITC-phalloidin in PBS and add the relevant secondary antibody dilution to identify the presence of your primary antibody. Pipette $200 \mu \mathrm{L}$ of the secondary antibody/phalloidin mix onto each of the coverslips. Cover the 24-well plate in tin foil for 30-60 min at room temperature to allow staining to take place. (see Note 16).

11. After 30-60 min remove the secondary antibody/phalloidin mix and wash twice with $500 \mu \mathrm{L}$ of PBS to remove excess phalloidin.

12. Now the slides are ready to mount. Place the appropriate amount of mounting medium (2-3 $\mu \mathrm{L}$ per coverslip) on each coverslip, remove the coverslips from the 24well plate with tweezers and place them on glass slides (see Note 17). Leave the slides overnight in the fridge covered in tin foil (see Note 18).

13. Image slides using either a conventional fluorescence or a confocal microscope with $63 \times$ or $100 \times$ oil immersion lenses.

\subsubsection{Post treatment of inhibitors spreading method}


This method allows the platelets to spread for a period of time before addition of the inhibitors.

1. Pipette $200 \mu \mathrm{L}$ of platelet suspension onto each coverslip. Place the lid on the 24well plate, and then place the 24 -well plate in the $37^{\circ} \mathrm{C}$ incubator for 25 min to allow platelets to adhere to the coverslips (see Note 19).

2. After 25 min take out the plate from the incubator and remove the solution. Then wash the slides twice with $500 \mu \mathrm{L}$ of PBS to remove any unbound platelets using a Pasteur pipette.

3. At this point add solutions of the relevant inhibitors (see Subheading 2.2 item 9) made up in Tyrode's buffer to the coverslips and then place back in the incubator for 10-60 $\min$.

4. After the relevant period of time remove the inhibitor solutions and wash twice with $500 \mu \mathrm{L}$ of PBS to remove any excess inhibitors.

5. Fix and stain as per the prior incubation method (from step 4 of Subheading 3.2.1).

\subsubsection{Image analysis}

To complete the relevant image analysis Image $\mathrm{J}$ is used. If Zeiss, Zen software is used to take the images, then the .czi files can be imported into ImageJ using the Bioformats plugin (see Note 20). The Bioformats plugin can read the .czi files, and will identify the relevant sizing information in the image. However if JPEG or TIFF files are imported it will be necessary to have the correct sizing information to put into image $\mathrm{J}$. In this case a graticule image will be require to identify how many pixels in the image correspond to a certain distance.

1. Adhesion analysis.

a. Take 5 random fields of view.

b. Count all the platelets present in the field of view. However these must be complete platelets. If you are only able to see half a platelet this does not count in the adhesion count.

c. The graph can then be standardized either to the control, or can be presented as the number of platelets per a defined area.

\section{Spreading analysis.}


a. Take 100 random platelets from across 5 fields of view.

b. Use the free draw tab on ImageJ to draw individually around each platelet. Then measure the area $(\mathrm{Ctrl}+\mathrm{m})$.

c. Average the 100 measurements

3. Stress fiber and actin nodule analysis.

a. Take 5 random fields of view.

b. Identify if a platelet contains actin nodules (see Figure 1) or stress fibers (see Figure 1). Some platelets may contain both.

c. The number of platelets containing either stress fibers or actin nodules is then divided by the number of platelets on that field of view to obtain a percentage. This is completed for all 5 fields of view.

4. Actin nodule analysis.

a. Take 5 random fields of view.

b. Identify if a platelet contains actin nodules (see Figure 1).

c. Count the number of actin nodules within each platelet.

d. Actin nodule size is determined through the circling of punctate areas of dense actin staining, previously identified as actin nodules, using ImageJ and the Bioformats plugin. 100 actin nodules in total are analyzed in actin nodule-positive, stress fiber-negative platelets and their size averaged.

All of these analyses will be completed on three separate experiments in order to be able to identify statistical significance.

\subsection{Platelet Spreading for Imaging Analysis (Real time)}

1. Prepare 24-well or 6-well glass bottomed dishes with the relevant matrix protein as per Subheading 3.2.

2. Resuspend washed platelets to $2 \times 10^{7} / \mathrm{mL}$ in Tyrode's buffer. Add $500 \mu \mathrm{L}$ or $2 \mathrm{~mL}$ of platelet suspension to each well of the 24-well or 6-well dishes respectively.

3. Monitor spreading using either DIC or fluorescence (if platelets express a fluorescent protein) microscopy for up to $1 \mathrm{~h}$ at $37^{\circ} \mathrm{C}$. 
4. Spread platelets can then be fixed, stained and imaged as per Subheading 3.2.1, from step 4.

\subsection{Platelet Spreading for Western Blotting and Pulldown Analysis}

The following protocol describes the preparation of lysates from spread platelets for western blotting or to determine the levels of activated Rho GTPases using pulldown assays. The procedure can be carried out in 6-well plates for western blotting or in 10-cm dishes for GTPase activation assays. Prepare the required number of wells or dishes depending on the experimental conditions and time points and the corresponding controls.

\subsubsection{Western blotting}

1. Coat 6-well plates by pipetting up to $1 \mathrm{~mL}$ of the matrix protein of choice onto the relevant well. Cover the 6 -well plate with the lid and incubate at $4^{\circ} \mathrm{C}$ overnight (See Note 13).

2. On the day of use prepare a minimum of $500 \mathrm{~mL}$ of PBS for washing and turn on incubator and set to $37^{\circ} \mathrm{C}$.

3. Remove the plates from the fridge, remove the matrix protein and wash twice with 1 $\mathrm{mL}$ of PBS to remove any unbound matrix protein. This can be done using a Pasteur pipette or aspiration device.

4. Prepare PBS-BSA and block the plates with $1 \mathrm{~mL}$ for $1 \mathrm{~h}$ at room temperature.

5. Remove the PBS-BSA solution and wash the plates twice with $1 \mathrm{~mL}$ of PBS to remove any unbound BSA using a Pasteur pipette or aspiration device.

6. At this point resuspend washed platelets to $2 \times 10^{8} / \mathrm{mL}$ in Tyrode's buffer. As per fixed spreading, two different methods for platelet spreading can be followed.

7. Prior incubation method: 
a. Add Rho GTPase inhibitors to the platelet suspension at the correct concentration (see Subheading 2.2 item 9). Preincubate the inhibitors with the platelets for 2 min prior to addition to the wells.

b. Pipette $1 \mathrm{~mL}$ of platelet suspension onto each well. Place the lid on the 6-well plate, and then place the 24 -well plate in the $37^{\circ} \mathrm{C}$ incubator for $45 \mathrm{~min}$ to allow platelets to adhere to the coverslips.

c. After 45 min take out the plate from the incubator and remove the solution. Proceed with step 9.

8. Post-spreading method:

a. Pipette $1 \mathrm{~mL}$ of platelet suspension onto each well. Place the lid on the 6-well plate, and then place the plate in the $37^{\circ} \mathrm{C}$ incubator for 25 min to allow platelets to adhere to the wells (see Note 19).

b. After 25 min take out the plate from the incubator and remove the solution. Then wash the wells twice with PBS to remove any unbound platelets using a Pasteur pipette or aspiration device.

c. At this point add solutions of the relevant inhibitors (see Subheading 2.2 item 9) made up in Tyrode's buffer to the wells and then place back in the incubator for $10-60 \mathrm{~min}$.

d. After the relevant period of time remove the inhibitor solutions and proceed with step 9.

9. Wash the wells with PBS. Place the plate on ice and add $100 \mu \mathrm{L}$ of lysis buffer. Use a cell scraper is used to ensure that all the platelets are removed from the surface of the well.

10. Transfer lysates to microcentrifuge tubes and either freeze down and store at $-20^{\circ} \mathrm{C}$ until needed or carry out a protein concentration.

11. Combine equal amounts of protein form the different conditions with $6 \times$ Laemmli buffer and boil for 5 min.

12. Resolve samples by SDS-PAGE (10\% gel) followed by Western blotting using standard techniques. Use RhoA and Rac1 specific antibodies to visualize total levels, 
phosphor-specific antibodies to monitor changes in the phosphorylation status and GAPDH as a loading control.

13. Image the blots using a fluorescence-based system to ensure linearity of the signals. As an example of this method Figure 2 shows the effect of $\mathrm{PGI}_{2}$ on the phosphorylation of RhoA. Figure $2 \mathrm{a}-\mathrm{d}$ shows that RhoA ${ }^{\text {Ser188 }}$ phosphorylation is elevated in the presence of $\mathrm{PGI}_{2}$ over basal, alongside confirmation of PKA activity via phosphorylation of VASP. The downstream marker of myosin light chain phosphorylation can then also be monitored to show the effect of the phosphorylation of RhoA. These effects driven by $\mathrm{PGI}_{2}$ can then be reversed in the presence of PKA inhibitors. These changes in phosphorylation levels can then be quantified using densitometry comparing their levels to the loading control GAPDH. The phosphorylation of RhoA ${ }^{\text {Ser188 }}$ is thought to correlate to decreased activity levels for the Rho GTPases (23).

\subsubsection{Rho GTPase pulldowns}

1. Using 10-cm plates, proceed as in Subheading 3.4.1. steps 1-9 (depending on the method) but using 3-mL volumes per plate.

2. At the relevant time point place the plates on ice and wash twice with PBS.

3. Lyse with the RhoA/Rac lysis buffer supplied in the RhoA/Rac pulldown kit (200 $\mu \mathrm{L}$ per plate) on ice. Use a cell scraper to ensure that all the platelets are removed from the surface of the plate. Transfer the lysates to microcentrifuge tubes.

4. From each individual lysate, $50 \mu \mathrm{L}$ is used for protein determination and for Western blotting for total RhoA/Rac1 protein (see Note 21).

5. Mix 200-300 $\mu \mathrm{g}$ of protein of the lysate with $50 \mu \mathrm{g}$ of Rhotekin-RBD-beads or PAKGST beads and incubate for 90 min on a rotator at $4^{\circ} \mathrm{C}$ (see Note 22).

6. Pellet the beads by centrifugation at maximum speed in a microcentrifuge for $1 \mathrm{~min}$ and remove $90 \%$ of the supernatant. 
7. Wash bead pellets with $400 \mu \mathrm{L}$ of wash buffer. Ensure the beads are fully resuspended in wash buffer (see Note 23).

8. Pellet the beads by centrifugation at maximum speed in a microcentrifuge for $1 \mathrm{~min}$.

9. Remove the supernatant, add $40 \mu \mathrm{L}$ of $2 \times$ Laemmli buffer and boil for $5 \mathrm{~min}$.

10. Resolve total lysate and active samples by SDS-PAGE (10\% gel) followed by Western blotting using standard techniques. Use anti RhoA or Rac1 primary antibodies followed by the appropriate secondary antibodies.

11. Image the blots using a fluorescence-based system to ensure linearity of the signals. Densitometries for the active and total RhoA/Rac1 are measured by drawing equal sized rectangles to the bands on the blots obtained. These values are converted into ratios by dividing the respective active RhoA/Rac1 to its total RhoA/Rac1 counterpart.

As an example of this method Figure 2e,f shows the effect of $\mathrm{PGI}_{2}$ on the activity of RhoA. RhoA activity is elevated in control spread platelets. This elevation is reduced in the presence of $\mathrm{PGI}_{2}$ and is then returned normal levels in the presence of PKA inhibitors (Figure $2 \mathrm{e})$. The fold change can be identified as the band density of the active RhoA compared to the band density of the total RhoA (Figure 2f).

\section{Notes}

1. OxLDL and $\mathrm{nLDL}$ are produced within house following published protocols (24-27).

2. Thrombin is bought from Sigma as a lyophilized powder. It is then reconstituted as per the manufacturer's instructions to a concentration of $10 \mathrm{U} / \mathrm{mL}$. This is then aliquoted in $10-\mu \mathrm{L}$ aliquots and stored frozen at $-20^{\circ} \mathrm{C}$. Once it is thawed it is used on the day and then discarded.

3. Prostacyclin is diluted in methanol as a $2 \mathrm{mM}$ solution. It is then stored as $2-\mu \mathrm{L}$ aliquots at $-20^{\circ} \mathrm{C}$. If required it is taken out of the freezer just before it is needed as it has a half life of $2 \mathrm{~min}$. Once used it is then discarded. 
4. Indomethacin stock is dissolved in DMSO at a concentration of $10 \mathrm{mM}$. It is then aliquoted in $5-\mu \mathrm{L}$ aliquots. DMSO can be used in platelet spreading up to a concentration of $0.1 \%$.

5. The denaturation process ensures that the platelets do not respond to the BSA. Therefore a BSA alone slide should have very little platelet adhesion and spreading. In addition the BSA can then be used to block the areas of the slide that are not covered by the extracellular matrix proteins that has been used.

6. Phalloidin is very toxic, may be fatal if swallowed, inhaled or absorbed through the skin. For personal protection, wear glasses and gloves. Avoid dispersal of material in the air or on working surfaces.

7. Ensure you have the appropriate permissions to withdraw and handle human blood according to your institutional guidelines and comply with health and safety requirements.

8. When suspending the pellet ensure that you pipette gently. The platelets should puff off the pellet. If you disturb large lumps of the pellet, you risk pre-activating the platelets as you resuspend them.

9. When using the Beckman Coulter counter dilute $2.5 \mu \mathrm{L}$ of platelet suspension in 10 $\mathrm{mL}$ of diluent, and then count. This will help to ensure that the machine is less likely to be blocked by excessive numbers of platelets.

10. Do not euthanize mice using cervical dislocation, as this will result in tissue factor release and activation of the coagulation system preventing removal of blood and isolation of platelets. We normally use suffocation by $\mathrm{CO}_{2}$.

11. Blood can also be collected by cardiac puncture, depending on the preferences and skills of the researcher. A good bleeding would yield up to $1 \mathrm{~mL}$ of blood from a single adult mouse.

12. When removing the PRP cut off the end of a $200-\mu \mathrm{L}$ pipette tip, and then take up as much of the PRP as possible including the buffy coat. However try to ensure that you do not take up red blood cells.

13. To ensure the wells stay wet, place a wet towel in the $6 / 24$-well plate to provide a humid environment.

14. At this point the slides can be placed into the fridge and stored for up to a week, before staining. However, staining is best if completed straight away. 
15. The length of time required to complete staining is antibody specific. For many antibodies $1 \mathrm{~h}$ at room temperature should be sufficient. However, it is sometimes necessary to incubate the primary antibody overnight at $4^{\circ} \mathrm{C}$ to obtain good staining.

16. The concentration and incubation time of the secondary antibody may also need optimizing. Too much or too long incubation of the secondary can lead to non-specific binding and high background staining.

17. When lifting out the coverslip use a $200-\mu \mathrm{L}$ pipette tip to help lift the coverslip up before picking it up with some tweezers. Ensure that care is taken, as it is easy to both break the coverslip and to drop it.

18. The slides can be left for up to 1 week if required. However it is always best to image the following day to maximize the quality of the fluorescence.

19. 25 min of spreading has been shown to allow a good number of platelets to adhere, and to have the vast majority of those platelets to be fully spread. This ensures that the experiment will be conducted on fully activated platelets. However longer time points can be chosen if required.

20. The Bioformats plugin can be downloaded from https://imagej.net/Bio-Formats. It can then be installed depending on the computer system used.

21. We use the protein determination kit from Cytoskeleton. It requires only $3 \mu \mathrm{L}$ of sample and can be read after 1 min of incubation. This improves the speed of the experiment, which is critical as Rho GTPases in their active form can easily breakdown into their inactive GDP-bound form.

22. If the protein concentration is too high, then there will be non-specific binding of RhoGDP or Rac-GDP to the beads.

23. Do not invert or flick the microcentrifuge tube, and ensure also that the bead suspension is not aspirated into the pipette tip. 


\section{References}

1. Aslan, J.E., and McCarty, O.J. (2013) Rho GTPases in platelet function. J Thromb Haemost 11, 35-46.

2. Hodge, R.G., and Ridley, A.J. (2016) Regulating Rho GTPases and their regulators. Nat Rev Mol Cell Biol 17, 496-510.

3. Akbar, H., Shang, X., Perveen, R., Berryman, M., Funk, K., Johnson, J.F., Tandon, N.N., and Zheng, Y. (2011) Gene targeting implicates Cdc42 GTPase in GPVI and non-GPVI mediated platelet filopodia formation, secretion and aggregation. PLoS One 6, e22117.

4. Pleines, I., Eckly, A., Elvers, M., Hagedorn, I., Eliautou, S., Bender, M., Wu, X., Lanza, F., Gachet, C., Brakebusch, C., and Nieswandt, B. (2010) Multiple alterations of platelet functions dominated by increased secretion in mice lacking Cdc42 in platelets. Blood 115, 3364-3373.

5. Goggs, R., Savage, J.S., Mellor, H., and Poole, A.W. (2013) The small GTPase Rif is dispensable for platelet filopodia generation in mice. PLoS One 8, e54663.

6. McCarty, O.J., Larson, M.K., Auger, J.M., Kalia, N., Atkinson, B.T., Pearce, A.C., Ruf, S., Henderson, R.B., Tybulewicz, V.L., Machesky, L.M., and Watson, S.P. (2005) Rac1 is essential for platelet lamellipodia formation and aggregate stability under flow. J Biol Chem 280, 39474-39484.

7. Aburima, A., Wraith, K.S., Raslan, Z., Law, R., Magwenzi, S., and Naseem, K.M. (2013) CAMP signaling regulates platelet myosin light chain (MLC) phosphorylation and shape change through targeting the RhoA-Rho kinase-MLC phosphatase signaling pathway. Blood 122, 3533-3545.

8. Calaminus, S.D., Auger, J.M., McCarty, O.J., Wakelam, M.J., Machesky, L.M., Watson, S.P. (2007) Myosinlla contractility is required for maintenance of platelet structure during spreading on collagen and contributes to thrombus stability. J Thromb Haemost 5, 21362145. 
9. Paul, B.Z., Daniel, .JL., and Kunapuli, S.P. (1999) Platelet shape change is mediated by both calcium-dependent and -independent signaling pathways. Role of p160 Rhoassociated coiled-coil-containing protein kinase in platelet shape change. J Biol Chem 274, 28293-28300.

10. Calaminus, S.D., Thomas, S., McCarty, O.J., Machesky, L.M., and Watson, S.P. (2008) Identification of a novel, actin-rich structure, the actin nodule, in the early stages of platelet spreading. J Thromb Haemost 6, 1944-1952.

11. Poulter, N.S., Pollitt, A.Y., Davies, A., Malinova, D., Nash, G.B., Hannon, M.J., Pikramenou, Z., Rappoport, J.Z., Hartwig, J.H., Owen, D.M., Thrasher, A.J., Watson, S.P., and Thomas, S.G. (2015) Platelet actin nodules are podosome-like structures dependent on Wiskott-Aldrich syndrome protein and ARP2/3 complex. Nat Commun 6, 7254.

12. Aslan, J.E., Tormoen, G.W., Loren, C.P., Pang, J., and McCarty, O.J. (2011) S6K1 and mTOR regulate Rac1-driven platelet activation and aggregation. Blood 118, 3129-3136.

13. Yusuf, M.Z., Raslan, Z., Atkinson, L., Aburima, A., Thomas, S.G., Naseem, K.M., and Calaminus, S.D.J. (2017) Prostacyclin reverses platelet stress fibre formation causing platelet aggregate instability. Sci Rep 7, 5582.

14. Thornber, K., McCarty, O.J., Watson, S.P., and Pears, C.J. (2006) Distinct but critical roles for integrin $\alpha_{11 b} \beta_{3}$ in platelet lamellipodia formation on fibrinogen, collagen-related peptide and thrombin. FEBS $J$ 273, 5032-5043.

15. Roberts, W., Magwenzi, S., Aburima, A., and Naseem, K.M. (2010) Thrombospondin-1 induces platelet activation through CD36-dependent inhibition of the cAMP/protein kinase A signaling cascade. Blood 116, 4297-4306.

16. Nergiz-Unal, R., Lamers, M.M., Van Kruchten, R., Luiken, J.J., Cosemans, J.M., Glatz, J.F., Kuijpers, M.H., and Heemskerk, J.W. (2011) Signaling role of CD36 in platelet activation and thrombus formation on immobilized thrombospondin or oxidized low-density lipoprotein. J Thromb Haemost 9, 1835-1846.

17. McCarty, O.J., Calaminus, S.D., Berndt, M.C., Machesky, L.M., and Watson, S.P. (2006) von Willebrand factor mediates platelet spreading through glycoprotein $\mathrm{Ib}$ and $\alpha_{1 \mathrm{lb}} \beta_{3}$ in the presence of botrocetin and ristocetin, respectively. J Thromb Haemost 4, 1367-1378. 
18. Inoue, O., Suzuki-Inoue, K., Dean, W.L., Frampton, J., and Watson, S.P. (2003) Integrin $\alpha_{2} \beta_{1}$ mediates outside-in regulation of platelet spreading on collagen through activation of Src kinases and PLC $\gamma 2$. J Cell Biol 160, 769-780.

19. Inoue, O., Suzuki-Inoue, K., McCarty, O.J., Moroi, M., Ruggeri, Z.M., Kunicki, T.J., Ozaki, Y., and Watson, S.P. (2006) Laminin stimulates spreading of platelets through integrin $\alpha_{6} \beta_{1}$-dependent activation of GPVI. Blood 107, 1405-1412.

20. Alshehri, O.M., Hughes, C.E., Montague, S., Watson, S.K., Frampton, J., Bender, M., and Watson, S.P. (2015) Fibrin activates GPVI in human and mouse platelets. Blood 126, 1601-1608.

21. Qiu, Y., Brown, A.C., Myers, D.R., Sakurai, Y., Mannino, R.G., Tran, R., Ahn, B., Hardy, E.T., Kee, M.F., Kumar, S., Bao, G., Barker, T.H., and Lam, W.A. (2014) Platelet mechanosensing of substrate stiffness during clot formation mediates adhesion, spreading, and activation. Proc Natl Acad Sci U S A 111, 14430-14435.

22. Kee, M.F., Myers, D.R., Sakurai, Y., Lam, W.A., and Qiu, Y. (2015) Platelet mechanosensing of collagen matrices. PLoS One 10, e0126624.

23. Faure, J., and Dagher, M.C. (2001) Interactions between Rho GTPases and Rho GDP dissociation inhibitor (Rho-GDI). Biochimie 83, 409-414.

24. Naseem, K.M., Goodall, A.H., and Bruckdorfer, K.R. (1997) Differential effects of native and oxidatively modified low-density lipoproteins on platelet function. Platelets 8, 163173.

25. Gerry, A.B., Satchell, L., and Leake, D.S. (2008) A novel method for production of lipid hydroperoxide- or oxysterol-rich low-density lipoprotein. Atherosclerosis 197, 579-587.

26. el-Saadani, M., Esterbauer, H., el-Sayed, M., Goher, M., Nassar, A.Y., and Jurgens, G. (1989) A spectrophotometric assay for lipid peroxides in serum lipoproteins using a commercially available reagent. J Lipid Res 30, 627-630.

27. Magwenzi, S., Woodward, C., Wraith, K,S., Aburima, A., Raslan, Z., Jones, H., McNeil, C., Wheatcroft, S., Yuldasheva, N., Febbriao, M., Kearney, M., and Naseem, K.M. (2015) Oxidized LDL activates blood platelets through CD36/NOX2-mediated inhibition of the cGMP/protein kinase G signaling cascade. Blood 125, 2693-2703. 


\section{Figure legends}

Figure 1: Representative images of filopodia, lamellipodia, actin nodule, and stress fiber formation in platelets. Platelets $\left(2 \times 10^{7} / \mathrm{mL}\right)$ were incubated for 45 min on coverslips coated with $100 \mu \mathrm{g} / \mathrm{mL}$ fibrinogen before fixation, staining with FITC-phalloidin, mounting, and imaging with a fluorescent microscope. Images are representative of (a) filopodia formation (red arrows) and actin nodules (yellow arrows); (b) lamellipodia formation (blue arrows); (c) stress fibers (green arrows).

Figure 2: $\mathrm{PGI}_{2}$ induces a PKA signaling response in spread platelets. (a) Platelets $\left(2 \times 10^{8} / \mathrm{mL}\right)$ were spread on $100 \mu \mathrm{g} / \mathrm{mL}$ fibrinogen for $25 \mathrm{~min}$ in the presence or absence of PKA inhibitors $100 \mu \mathrm{M}$ RP-8CPT-CAMP (RP) and $2 \mu \mathrm{M}$ KT5720 (KT), before being washed with PBS. The platelets were then treated with Tyrode's buffer containing $10 \mathrm{nM} \mathrm{PGI}_{2}$ with or without PKA inhibitors (100 $\mu \mathrm{M}$ RP-8CPT-cAMP and $2 \mu \mathrm{M}$ KT5720), or $1 \mu \mathrm{M}$ forskolin, or $10 \mu \mathrm{M}$ Y27632, for a further $10 \mathrm{~min}$. The samples were then lysed with Laemmli buffer before being western blotted for pVASP ${ }^{\text {Ser159, }}$, pMLC ${ }^{\text {Ser19 }}, \mathrm{pRhoA}^{\text {Ser188, }}$, and GAPDH. Cropped gel Images are representative of at least three experiments. (b-d) Densitometry for the western blots; pVASP ${ }^{\text {ser159 }}$, pMLC ${ }^{\text {ser19 }}$ and pRhoA $^{\text {ser188 }}$ using GAPDH as the loading control. The ratios were standardized to the control. (e) Spread platelets as in (a) were treated with Tyrode's buffer containing $10 \mathrm{nM} \mathrm{PGI}_{2}$ with or without PKA inhibitors $100 \mu \mathrm{M}$ RP-8CPT-CAMP and $2 \mu \mathrm{M} \mathrm{KT5720} \mathrm{for} \mathrm{a} \mathrm{further} 10 \mathrm{~min}$. The samples were then lysed before the addition of Rhotekin-GST beads. Samples were then western blotted for active RhoA and total RhoA. Cropped gel Images are representative of at least three experiments. (f) Images of RhoA pull down were analyzed for densitometry. Analysis is an average of at least $n=3$ experiments. ${ }^{*} p<0.05$ (ANOVA). Reproduced from ref. 13. 